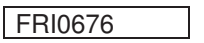

RESPONSIVENESS OF PATIENT REPORTED OUTCOMES MEASUREMENT INFORMATION SYSTEM (PROMIS $^{\circledR}$ ) COMPUTERIZED ADAPTIVE TESTS (CATS) IN SYSTEMIC LUPUS ERYTHEMATOSUS (SLE)

S. Kasturi ${ }^{1}$, J. Szymonifka ${ }^{2}$, J. Berman², K. Kirou², A. Levine ${ }^{2}$, L. Sammaritano ${ }^{2}$, L. A. Mandl ${ }^{2} .{ }^{1}$ Medicine/Rheumatology, Tufts Medical Center, Boston, ${ }^{2}$ Medicine/ Rheumatology, Hospital for Special Surgery, New York, United States

Background: The accurate measurement of patient reported outcomes is a priority for patient-centered care in SLE, a chronic systemic disease with significant impact on quality of life. PROMIS CATs are precise measures of physical, mental, and social health with construct validity in SLE. The longitudinal responsiveness (sensitivity to change) of PROMIS CATs in SLE patients is unknown.

Objectives: To evaluate the responsiveness of PROMIS CATs in SLE outpatients using patient and physician-derived anchors.

Methods: Adult SLE patients were recruited from an SLE Center of Excellence. Subjects completed 14 selected PROMIS CATs at two visits a minimum of one month apart. SLE disease activity was measured with a patient global assessment of change, a physician global assessment and the physician-derived SELENASLEDAI. Responsiveness of PROMIS scores was evaluated using known-groups validity. Effect sizes were compared across groups of patients who differed in their patient global assessment of change, physician global assessment, and SELENA-SLEDAl using Wilcoxon rank-sum tests.

Results: A diverse cohort of 228 SLE patients, including 45 (19.8\%) patients flaring by SELENA-SLEDAl, completed baseline surveys. Follow up surveys were completed by 190 (83\%). There was poor agreement between patient and physician global assessments (weighted kappa statistic [95\% Cl] $=0.16[0.04-0.28]$. Using the patient-based anchor, Anger, Pain Interference, and Physical Function CATs showed low to moderate responsiveness (table 1). Using the physician global assessment, only Anxiety CAT showed low to moderate responsiveness (effect size $-0.27,-0.17$, and $0.06[p=0.03]$ with $\geq 0.5$ point decrease, $<0.5$ point change, and $\geq 0.5$ point increase respectively), while with the SELENA-SLEDAI as anchor, only Applied Cognition-Abilities CAT showed responsiveness (0.34, $0.01,0.0[\mathrm{p}<0.01]$ with $\geq 3$ point decrease, $<3$ point change, and $\geq 3$ point increase respectively)

\begin{tabular}{lllll}
\hline PROMIS CAT & \multicolumn{3}{l}{ Patient Global Assessment of Change } & $\begin{array}{l}\text { p- } \\
\text { value }\end{array}$ \\
\hline & \multicolumn{3}{l}{ Better } \\
$(\mathbf{n = 7 4 )}$ & $\begin{array}{l}\text { Same } \\
(\mathbf{n}=79)\end{array}$ & $\begin{array}{l}\text { Worse } \\
(\mathbf{n}=\mathbf{3 3})\end{array}$ & \\
\hline Ability to Participate in Social Roles & 0.14 & 0.00 & 0.00 & 0.19 \\
Anger & $-\mathbf{0 . 3 7}$ & $-\mathbf{0 . 0 8}$ & $\mathbf{0 . 0 0}$ & $\mathbf{0 . 0 3}$ \\
Anxiety & -0.38 & -0.04 & -0.02 & 0.13 \\
Applied Cognition-Abilities & 0.15 & 0.00 & -0.03 & 0.30 \\
Applied Cognition-General & -0.08 & 0.00 & -0.06 & 0.47 \\
Concerns & & & & \\
Depression & -0.15 & 0.00 & 0.36 & 0.15 \\
Fatigue & -0.16 & 0.00 & -0.15 & 0.51 \\
Mobility & 0.14 & 0.00 & 0.00 & 0.26 \\
Pain Behavior & -0.14 & 0.00 & 0.10 & 0.07 \\
Pain Interference & $\mathbf{0 . 0 0}$ & $\mathbf{0 . 0 0}$ & $\mathbf{0 . 2 5}$ & $\mathbf{0 . 0 2}$ \\
Physical Function & $\mathbf{0 . 1 5}$ & $\mathbf{0 . 0 1}$ & $-\mathbf{0 . 1 4}$ & $\mathbf{0 . 0 2}$ \\
Satisfaction with Social Roles & 0.18 & 0.00 & 0.00 & 0.21 \\
\& Activities & & & & \\
Sleep Disturbance & 0.00 & -0.14 & -0.04 & 0.50 \\
Sleep-Related Impairment & -0.07 & 0.00 & -0.02 & 0.95 \\
\hline
\end{tabular}

Conclusions: PROMIS CATs showed modest responsiveness to patientreported, but generally not physician-derived changes in lupus health status in domains of anger, pain interference, and physical function. These data suggest that certain PROMIS CATs are precise and sensitive tools which may be used to measure and monitor important aspects of the patient experience of lupus not captured by physician-derived metrics. Further studies are needed to evaluate the responsiveness of PROMIS CATs in populations with greater SLE disease activity and more regular follow up.

Acknowledgements: Funding was provided by the Rheumatology Research Foundation Scientist Development Award.

Disclosure of Interest: None declared

DOI: 10.1136/annrheumdis-2018-eular.3152

\section{FRI0677 PREVALENCE AND SEROLOGICAL PROFILE OF ANTI- DFS7O POSITIVE SUBJECTS: DATA FROM A ROUTINE ANA COHORT}

T. Carbone $^{1,2}$, V. Pafundi ${ }^{2}$, G. Tramontano ${ }^{1}$, M. Gilio $^{1}$, C. Esposito ${ }^{1}$, M. C. Padula ${ }^{1}$, A. A. Padula ${ }^{1}$, S. D'Angelo ${ }^{1} .{ }^{1}$ Rheumatology Institute of Lucania - IReL,

${ }^{2}$ Immunopathology Laboratory, San Carlo Hospital, Potenza, Italy

Background: Anti-Dense Fine Speckled 70 (DFS70) antibodies are a common finding in clinical laboratory referrals. High prevalence of DFS70 autoantibodies in healthy population and usual negative association with Antinuclear Antibody (ANA)-associated autoimmune rheumatic diseases were reported.

Objectives: The aim of this study was to evaluate the prevalence of anti-DFS70 antibodies in a routine diagnostic laboratory setting and their association with other circulating serum autoantibodies.

Methods: Consecutive sera submitted for ANA screening were analyzed for anti-DFS70 antibodies by indirect immunofluorescence (IIF) ( $n=3175,1030$ men and 2145 women) then confirmed by Immunoblotting. IIF DFS70 positive adult patients were recruited previous written consent and tested for the following autoantibodies: anti-ENA, anti-dsDNA, -anti-TPO, anti-TG, anti-tTg, aCL, anti-PCA, AMA, ASMA, anti-LKM, anti-MPO, anti-PR3 and ASCAs.

Results: The prevalence of anti-DFS70 antibodies was $1.7 \%(n=55)$ in the whole population and $4.6 \%$ in the ANA-positive samples. Comparison between DFS70 IIF and Immunoblotting showed an excellent correlation between the two methods $(\mathrm{R}=0.99)$. Analysis of anti-DFS70 antibodies titers distribution revealed that $63 \%$ of the total cohort showed high titers ( $\left.{ }^{3} 1: 640\right)$. Gender difference (female: male, 4:1) was observed in anti-DFS70 positive group and in anti-DFS70 negative/ANA positive group. The prevalence of anti-DFS70 positive female $(2.1 \%, 45$ $2145)$ was statistically significant higher than males $(1.0 \%, 10 / 1030)(p<0.05)$ The comparison among referring sources evidenced a prevalence of anti-DFS70 positive subjects from Endocrinology Department $(9.1 \%$ versus $2.6 \%$ from Haematology, $2.1 \%$ from outpatients, $1.6 \%$ from Neurology, $1.2 \%$ from Internal Medicine, $1 \%$ from Cardiology, $0.6 \%$ from Rheumatology). Of note, our data evidenced isolated reactivity of anti-DFS70 autoantibodies in males group, while $51 \%$ of females showed concomitant disease-marker autoantibodies.

Conclusions: We found a prevalence of anti-DFS70 antibodies in adult sera from routine ANA cohort of $1.7 \%$. The serological profile of DFS70-positive females required further investigations in order to define the presence of serum autoantibodies. Anti-DFS70 reactivity in male population may represent an exclusive biomarker predicting the absence of other autoantibodies.

Acknowledgements: The authours would like to express their expecial appreciation and thanks to Prof. Ignazio Olivieri who died on July 28th, 2017. He was an example of strenght and tenacity with a contagious enthusiasm for a rigorous scientific research.

Disclosure of Interest: None declared

DOI: 10.1136/annrheumdis-2018-eular.6476

\section{FRI0678 VALIDITY OF THREE 0-10 VISUAL ANALOG SCALES (VAS) FOR QUANTITATIVE PHYSICIAN ASSESSMENT OF INFLAMMATION, DAMAGE, AND DISTRESS TO SUPPLEMENT A PHYSICIAN GLOBAL ASSESSMENT 0- 10 VAS}

\section{T. Pincus ${ }^{1}$, I. Castrejon ${ }^{1}$, J. A. Block ${ }^{1} .{ }^{1}$ Rheumatology, Rush University Medical} Center, Chicago, United States

Background: Rheumatologists generally view their primary goal as control of inflammation in order to prevent long-term damage, and quantitative assessment involves measures of inflammatory activity (lab tests, joint counts, and indices). Although structural damage and patient distress (fibromyalgia, depression, etc.) are widely recognized, these problems generally are described narratively, and not assessed quantitatively. Recent advances in control of inflammation, as well as increased degenerative diseases in an aging population and recognition of a high prevalence of fibromyalgia, may have shifted rheumatologists' patient mix more prominently toward damage and distress vs inflammation.

Objectives: To analyze physician global assessment (DOCGL) on a 0-10 visua analog scales (VAS), and 3 additional 0-10 VAS for inflammation, damage, and distress, as well as estimates of the proportion of each to explain DOCGL.

Methods: Rheumatologists at one academic site complete a 0-10 DOCGL VAS, 3 further 0-10 VAS to assess inflammation (reversible disease) (DOCINF), join and other organ damage (irreversible disease) (DOCDAM), and patient distress (fibromyalgia, depression), etc. (DOCSTR), in routine care. The proportion of DOCGL attributed to inflammation, damage, and distress (total $=100 \%$ ) also is estimated. Mean values were analyzed in a cross-sectional study of 570 patients, and compared in subgroups with rheumatoid arthritis (RA), osteoarthritis $(O A)$, or fibromyalgia (FM), using t tests and analysis of variance (ANOVA).

Results: Mean DOCGL VAS was 4.4/10 in all patients, 4.4 in 131 with OA, 4.6 in 98 with RA, and 5.2 in 89 with FM (table 1). Highest mean scores were seen for DOCINF in RA, DOCDAM in OA, and DOCSTR in FM $(p<0.001)$, indicating face validity. Nonetheless, mean DOCDAM was higher than DOCINF in all groups, including RA, and mean estimates of the proportion of DOCGL attributed to damage was greater than to inflammation in all groups (table 1). Scores for DOCSTR were higher than for DOCINF in all groups other than RA. 